\title{
Ethical issues in placebo-controlled trials of COVID-19 vaccines
}

\author{
Questões éticas em ensaios clínicos controlados \\ com placebo, de vacinas contra COVID-19
}

\author{
Cuestiones éticas en los ensayos controlados con \\ placebo para las vacunas de COVID-19
}

\author{
Ana Cecilia Amado Xavier de Oliveira 1 \\ Francisco José Roma Paumgartten 1
}

doi: 10.1590/0102-311X00007221
Whenever there are effective and safe therapeutic or prophylatic interventions for COVID-19, the use of placebos instead of active comparators in randomized clinical trials is a delicate ethical issue.

In the exercise of the profession, physicians are morally committed to offer their patients the therapeutic or prophylactic interventions they deem the best. When doctors are clinical investigators and randomly assign patients to receive test treatments or inactive (placebo) comparators - if effective therapeutic or prophylatic interventions exist - they break their professional commitment and Hippocratic Oath ("the health of my patient will be my first consideration"). Furthermore, offering placebos when there are efficacious drugs or vaccines violates the equipoise ("equilibrium state") principle, which many doctors believe to be an essential requirement to render randomized trials ethically. This principle states that physicians/researchers must be in a state of genuine uncertainty about the relative merits (efficacy/safety) of the alternative interventions being tested 1 .

As clinical investigators, physicians are aware that - even if effective treatments exist for a condition being studied - for scientific reasons, the use of an inactive comparator may have methodological advantages. Not surprisingly, placebo use in randomized trials is a disputed subject. The updated versions of international codes of medical ethics explicitly state that inactive comparators and/or no intervention are acceptable only if (1) there is no proven effective intervention for the condition under investigation, (2) not to treat poses negligible risks for participants, (3) there are compelling methodological reasons for using placebo, and not to treat does not imply in risk of serious harm to participants.

Between 1976 and 2004, the World Medical Association's Declaration of Helsinki forbade the use of placebo in clinical trials if a proven effective (prophylatic, therapeutic, or diagnostic) intervention exists. As of 2008, the prohibition was eased and using placebo instead of active comparator was considered acceptable: "Where for compelling and scientifically sound methodological reasons the use of placebo is necessary to determine the efficacy or safety of an intervention and the patients who receive placebo or no treatment will not be subject to any risk of serious or irreversible harm" 2 . The most recent Declaration of Helsinki (2008 and 2013) 2 revisions remarked that "Extreme care must be taken to avoid abuse of this option".

In this regard, the latest version of the Brazilian Code of Medical Ethics (CME) (Resolution n. 2,217/2018 from Brazilian Federal Council of Medicine) 3 (p. 42) is even more restrictive, stating that: It is forbidden for physicians: "Art. 106. To maintain any type of connection with clinical studies in humans
1 Escola Nacional de Saúde Pública Sergio Arouca, Fundação Oswaldo Cruz, Rio de Janeiro, Brasil.

\section{Correspondence}

F. J. R. Paumgartten Laboratório de Toxicologia Ambiental, Escola Nacional de Saúde Pública Sergio Arouca, Fundação Oswaldo Cruz. Av. Brasil 4036, Rio de Janeiro, $R J$ 21040-361, Brasil. paum@ensp.fiocruz.br 
using a placebo as the sole medical intervention, whenever there is an effective prophylactic or therapeutic measure available".

In a fast response of science to a respiratory virus challenging pandemic, candidate COVID-19 vaccines were developed using traditional (inactivated viruses) and innovative biotechnological approaches (mRNA and modified viral vectors). Dozens of candidate vaccines are now being clinically tested in phase 2 (safety and immunogenic response) and phase 3 (safety and efficacy) trials. As of January 2021, some COVID-19 vaccines were demonstrated to be safe and highly effective, and subsequently approved for emergency use and/or received a final regulatory approval. Currently, several recognized efficacious vaccines are being used in mass vaccination campaigns worldwide.

Phase 3 vaccine trials can only be carried out in places where transmission rates are high, so that a target minimum number of infected people is attained allowing a statistically robust assessment of predefined primary efficacy endpoints in a reasonable time interval. When this target number of infected participants is reached, investigators proceed to interim analysis of efficacy data. In practice, to measure vaccine efficacy (VE), researchers calculate infection incidence rates (IR) among all vaccinated and unvaccinated (placebo-recipients) participants and determine the percentage reduction in IR among vaccinated (VIR) compared to IR in unvaccinated (UIR) participants, i.e. 4:

$$
\mathrm{VE}(\%)=[(\mathrm{UIR}-\mathrm{VIR}) / \mathrm{UIR}] \times 100
$$

Although this data evaluation is robust enough to demonstrate that vaccines are safe and confer protection against SARS-CoV-2 infection, and/or attenuates the disease severity, it does not inform how long the protection will last. On November 27, 2020, the European Medicine Agency (EMA) 5 warned COVID-19 vaccine investigators and regulators that the trials should continue (followup period) beyond "the time when the pre-defined cases of COVID-19 disease for final analysis in a trial have been reached" to provide "more precise information on longer-term safety and efficacy". Once some COVID-19 vaccines had proven to be efficacious (phase 3), subsequent placebo-controlled RCTs and/ or follow-up study poses ethical dilemmas for researchers 5 .

If a candidate vaccine proves to be efficacious, placebo-recipients should be informed that they remain unprotected. Moreover, it should be offered to them vaccination if they want to. This moral obligation stems from beneficence (serve the best interests of trial participants) and non-maleficence (to do no harm or allow harm to be caused to a participant by neglect) ethical principles applicable to RCTs. Vaccination of placebo-recipients before completion of the follow-up period, on the other hand, jeopardizes the investigation of long-term efficacy and safety, such as recommended by EMA.

Another ethical dilemma refers to placebo-controlled RCT of new candidate vaccines after efficacious COVID-19 vaccines are already in use. Randomized placebo-controlled trials with relevant primary efficacy endpoints and robust sample sizes are study designs that provide robust, fast, and straightforward evidence of vaccine efficacy. Redesigning vaccine trials to use active instead of inactive (placebo) comparators - or to conduct comparative trials -, is likely to require larger sample sizes, longer study duration, and may not be feasible.

\section{Vaccination of placebo-recipients after final analysis}

The easiest way to resolve uncertainties regarding how long protection lasts and long-term safety is to continue placebo-controlled trials beyond the time when the target number of infected participants is attained for final analysis. Otherwise, researchers must rely on large observational field studies of vaccine effectiveness.

Pfizer-BioNtech and Moderna COVID-19 (m-RNA-based) vaccines were announced to be 90-95\% effective in phase 3 and both companies recognized their ethical obligation to offer effective vaccines "as soon as possible" to those who had been placebo recipients in their studies 6,7,8. Of course, complying with this unquestionable ethical obligation implies in loss of follow up data about longterm efficacy and safety, as previously explained. Moreover, because of scarcity of vaccines to attend the US immunization plan, the placebo recipients would receive vaccine even when they have not been prioritized for vaccination outside the trial, decreasing immunization plan health equity gains 6 . 
AstraZeneca (viral vector, non-replicating) and CoronaVac (inactivated virus) COVID-19 vaccines, whose phase 3 trials occurred in Brazil, proved to be safe and effective, and are planned to be soon delivered to use through Brazil's National Immunization Programme (PNI). In contrast to Pfizer's vaccine trial, both AstraZeneca and CoronaVac Brazilian phase 3 trials enrolled only health professionals (Identifiers: NCT04456595, NCT04536051. http://www.ClinicalTrials.gov, accessed on 10/Jan/2021), who belong to the top priority group for COVID-19 vaccination according to the announced country's immunization plan. However, as far as we are aware, none of the vaccine research teams informed whether placebo recipients in their trials will receive the active vaccine before completion of the planned 12 month post-final vaccination follow-up.

The AstraZeneca trial used a meningococcal vaccine (MenACWY) as placebo control for COVID-19 vaccine, and so all study participants received paracetamol to prevent fever. This "active" placebo was used to ensure a better masking (participant single-blinded study); however, concomitant administration of paracetamol may have concealed some minor acute side effects common to most vaccines (local pain, fever) 9 .

\section{Further trials of COVID-19 vaccine candidates}

Up to January 11, 2021, World Health Organization lists 172 candidate COVID-19 vaccines in preclinical, and 63 in clinical development stages. Some vaccines in more advanced stage of clinical development have been recently demonstrated to be safe and efficacious in placebo-controlled RCTs 10,11. This vaccine development landscape suggests that more COVID-19 vaccine candidates should enter phase 3 testing in the next few months. To be ethical, further randomized trials must assign participants to active control arms (efficacious vaccines), not to placebo control arms.

\section{Facing the ethical dilemma}

If effective and safe interventions exist, placebo-controlled RCTs are forbidden by codes of medical ethics, unless there are compelling and scientifically sound reasons for placebo use and, even more significant, "if patients who receive placebo will not be subject to any risk of serious or irreversible harm". Currently, not protecting a group of trial participants with available efficacious vaccines is to subject them -, especially those who belong to a high risk group - to serious and irreversible harm, including death risk.

Thus, core ethical values strongly recommend that participants who had received placebo in COVID-19 vaccine trials should be promptly vaccinated after an interim analysis had proven that the vaccines are efficacious, even though this may jeopardize the study follow-up for long-term efficacy and safety data. How long protection lasts, and long-term safety could be otherwise investigated by observational (field) studies of vaccination effectiveness. Another ethics-compliant approach is to vaccinate at least the placebo recipients belonging to groups (older adults and other groups) at higher risk of severe disease, while the remaining lower risk ones are followed-up for long-term outcomes.

Similarly, further RCTs of COVID-19 vaccines should be designed to compare their efficacy/ safety with those of vaccines already in use, i.e., the study-design must involve active (proven effective vaccines) instead of inactive (placebo) comparators. 


\section{Contributors}

A. C. A. X. Oliveira and F. J. R. Paumgartten participated with substantial contributions to the conception and design of the work, as well as on the acquisition, analysis, and interpretation of data, drafted the work and revised it critically and approval of the final version to be published.

\section{References}

1. Freedman B. Equipoise and the ethics of clinical research. N Engl J Med 1987; 317:141-5.

2. World Medical Association. WMA Declaration of Helsinki: ethical principles for medical research involving human subjects. https:// www.wma.net/policies-post/wma-declarationof-helsinki-ethical-principles-for-medical-re search-involving-human-subjects/ (accessed on 10/Jan/2021).

3. Conselho Federal de Medicina. Código de Ética Médica: Resolução CFM no 2.217, de 27 de setembro de 2018, modificada pelas Resoluções CFM no 2.222/2018 e 2.226/2019. Brasília: Conselho Federal de Medicina; 2019.

4. Orenstein WA, Bernier RH, Dondero TJ, Hinman AR, Marks JS, Bart KJ, et al. Field evaluation of vaccine efficacy. Bull World Health Organ 1985; 63:1055-6.

5. European Medicines Agency. Global regulators urge continuation of COVID-19 vaccine trials for long-term safety and efficacy follow-up. http://www.ema.europa.eu/en/news/globalregulators-urge-continuation-covid-19-vac cine-trials-longer-term-safety-eff (accessed on 10/Jan/2021).

6. Rid A, Lipsitch M, Miller FG. The ethics of continuing placebo in SARS-CoV-2 vaccine trials. JAMA 2021; 325:219-20.

\section{Additional informations}

ORCID: Ana Cecilia Amado Xavier de Oliveira (0000-0002-5798-2258); Francisco José Roma Paumgartten (0000-0002-6207-0149).
7. Wendler D, Ochoa J, Millum J, Grady C, Taylor HA. COVID-19 vaccine trial ethics once we have efficacious vaccines. Science 2020; 370:1277-9.

8. Polack FP, Thomas SJ, Kitchin N, Absalon J, Gurtman A, Lockhart S, et al. Safety and efficacy of the BNT162b2 mRNA COVID-19 vaccine. N Engl J Med 2020; 383:2603-15.

9. Ramasamy MN, Minassian AM, Ewer KJ, Flaxman AL, Folegatti PM, Owens DR, et al. Safety and immunogenicity of ChAdOx $1 \mathrm{nCoV}-19$ vaccine administered in a prime-boost regimen in young and old adults (COV002): a singleblind, randomised, controlled, phase $2 / 3$ trial. Lancet 2021; 396:1979-93.

10. Corum J, Wee SL, Zimmer C. Coronavirus vaccine tracker (2020). The New York Times 2020; 14 oct. https://www.nytimes.com/interac tive/2020/science/coronavirus-vaccine-track er.html.

11. World Health Organization. The COVID-19 candidate vaccine landscape. http://www.who. int/publications/m/item/draft-landscape-ofcovid-19-candidate-vaccines (accessed on 10/ Jan/2021). 